\title{
ENCAPSULATION OF ROXITHROMYCIN INTO GELLAN GUM MATRICES AND THE IMPACT OF OTHER NATURAL POLYMERS ON DRUG RELEASE
}

\author{
PIOTR GADZIŃSKI ${ }^{1}$, ANNA FROELICH ${ }^{1}$, MARCIN SOBÓL ${ }^{2}$, \\ URSZULA KOWALSKA ${ }^{2}$, MIROSŁAW SZYBOWICZ ${ }^{3}$ and TOMASZ OSMAŁEK ${ }^{1 *}$ \\ 'Department of Pharmaceutical Technology, \\ Poznan University of Medical Sciences (PUMS), Poznań, Poland \\ ${ }^{2}$ Center of Bioimmobilisation and Innovative Packaging Materials, \\ West Pomeranian University of Technology, Szczecin, Poland \\ ${ }^{3}$ Faculty of Technical Physics Poznan University of Technology, Poznań, Poland
}

\begin{abstract}
The aim of the work was to investigate the properties of polysaccharide matrices loaded with roxithromycin (ROX), made on the basis of low-acyl gellan and its blends with sodium alginate, pectin, karaya gum, methylcellulose, and $\kappa$-carrageenan. The obtained formulations were investigated as potential oral dosage forms with the ability to protect the drug from the acidic conditions of the stomach. Another desired feature of the obtained systems was the sustained release of the active ingredient allowing for potential shifting of the therapeutic effect to the colon. The morphology of the matrices was evaluated with optical and scanning electron microscopy. Moreover, Raman spectroscopy and thermal analysis were performed for ROX, polymers, ROX/polymers physical mixtures and the matrices. Next, the swelling behavior was examined. The matrices were evaluated for ROX content and encapsulation efficiency. The last stage concerned the drug release studies. All matrices after production revealed more or less oval shape with visible deformation most probably occurring during drying. Raman analysis and DSC confirmed the crystalline form of ROX and showed no evidence of interactions between the drug and the excipients. It was shown that the matrices containing gellan combined with methylcellulose or $\kappa$-carrageenan at $\mathrm{pH}=7.4$ released $\mathrm{ROX}$ slower than the other matrices which might be promising in terms of colonic drug delivery. Moreover, the polymer matrices remained physically stable at acidic $\mathrm{pH}$ similar to the environment of the stomach. However, in these conditions drug degradation was observed which indicates the necessity to further modify the applied technology.
\end{abstract}

Keywords: roxithromycin, gellan gum, natural polymers, ionotropic gelation, drug release

Undoubtedly, the development of pharmaceutical technology would not be possible without the presence of naturally derived polymers. Among their most beneficial features, one can mention biocompatibility, ability to degrade in the body, lack or very low toxicity or relatively low production costs $(1,2)$. These compounds have been used both in the technology of traditional drug dosage forms and advanced innovative drug delivery systems $(3,4)$. It is well known that besides many different factors, the therapeutic efficiency of various active substances is related also to their stability in the acidic environment of the stomach $(5,6)$. In the case of acid-sensitive compounds, the most common reactions that leading to loss of activity are hydrolysis and isomerization $(7,8)$. Groups of compounds widely described as labile at low $\mathrm{pH}$ are macrolide antibiotics (erythromycin, roxithromycin) (9) and proton pump inhibitors, e.g. omeprazole (10). The problem can be partially solved by the simultaneous administration of antacids. However, probably the most popular methods for drug protection involve the application of polymeric coatings or carriers that release the drug in distal parts of the gastrointestinal tract $(11,12)$. In this case, $\mathrm{pH}$-sensitive synthetic polymers are frequently used (13). Another possible way to modify the drug release after oral administration is encapsulation of the API into polysaccharide matrices obtained by means of ionotropic gelation (14-16). One of the examples widely investigat-

* Corresponding author: e-mail: tosmalek@ump.edu.pl 
ed for such purpose is gellan gum, a microbial polymer (17). The matrices based on gellan reveal the $\mathrm{pH}-$ related properties, which means that at low $\mathrm{pH}$ they are stable but start to absorb water, decompose and release the drug as the $\mathrm{pH}$ increases. The $\mathrm{pH}-$ dependent behavior of gellan matrices can be potentially used for the protection of drugs that degrade in the presence of acidic stomach fluid $(18,19)$. The properties of gellan matrices and their behavior after oral administration can be easily and distinctly modified by various factors including the composition of the initial mixture. The concentration of gellan is a very important factor affecting the properties of the final product. However, the amount and type of additional polymers, as well as the presence of other components, like surfactants, oils or fillers, should also be taken into consideration as the elements with the potential impact for the drug release process. Another way to change the properties of the matrices is the modification of the production process parameters, including the temperature of the initial mixture and gelling solution, type and concentration of the gelling ions, mixing speed, instillation height or curing time after gelling, etc. (20-22).

Roxithromycin (ROX) is a II generation macrolide antibiotic. Structurally it is a semi-synthetic derivative of erythromycin, with the molecular formula of $\mathrm{C}_{41} \mathrm{H}_{76} \mathrm{~N}_{2} \mathrm{O}_{15}$ with the molecular weight of $837.058 \mathrm{~g} / \mathrm{mol}$. The molecule of ROX (Fig. 1) contains additional $\mathrm{N}$-oxime chain attached to the lactone ring. ROX reveals very low solubility in water $(0.0189$ $\mathrm{mg} / \mathrm{L}$ at $25^{\circ} \mathrm{C}$ ) and its octanol/water partition coefficient is equal to $1.7(12,23)$. As all macrolides, ROX reveals bacteriostatic action by attaching to $50 \mathrm{~S}$ ribosome subunit which disables peptides translocation and inhibits microbial protein synthesis pathway. Moreover, the drug very efficiently penetrates multinucleated leucocytes and macrophages and improves their

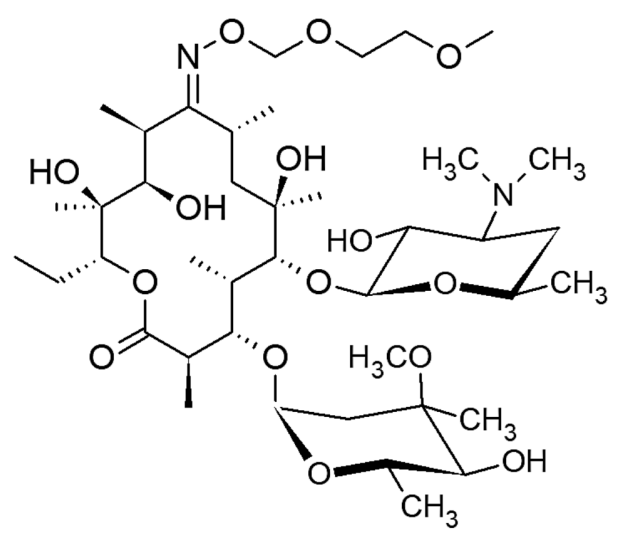

Figure 1. The molecular structure of roxithromycin. chemotactic and adhesive properties, which results in escalation of phagocytosis and bacterial lysis. ROX is mostly prescribed in the treatment of mild or moderate infections including acute pharyngitis, acute tonsillitis and impetigo $(12,23,24)$. Unfortunately, some reports indicate that besides very fast $\left(\mathrm{T}_{\max } \sim 2 \mathrm{~h}\right)$ and efficient (72-85\%) absorption from the gastrointestinal tract (GIT), ROX is very unstable in the acidic environment of the gastric fluid. The inactivation process is rapid and according to Ostrowski et al., after $15 \mathrm{~min}$ no more than $5.2 \%$ of the initial dose remains at the $\mathrm{pH}$ of 1.2. The experiments conducted simultaneously at $\mathrm{pH}=6.8$ did not reveal any significant changes in the drug concentration (9). The dominating process which leads to the deactivation of ROX is $\mathrm{pH}$-dependent transformation to Z-isomer. Moreover, the drug undergoes such reactions as hydrolysis, demethylation, dealkylation or photodegradation, when subjected to light. Further analysis confirmed that in most cases the L-cladinose moiety is responsible for the $\mathrm{pH}$-related deactivation $(25,26)$. The topic has interest and some attempts have been already made to protect ROX against stomach fluid and modify its release by shifting it to distal parts of the GIT. For example, Koopaei et al. showed that the incorporation of ROX to nanocarriers results in the stability increase. The authors prepared ROX-loaded nanoparticles with pegylated poly(lactide-co-glycolide) (27).

Some studies indicate that ROX apart from typical antimicrobial activity reveals also some antiinflammatory and immunoregulatory properties, which might be useful also in the therapy of inflammatory bowel disease $(28,29)$. Ahmad et al. revealed that beneficial effects of ROX administered to mice as a pretreatment before the induction of colitis were comparable to mesalazine, a therapeutic agent commonly applied in ulcerative colitis, while diclofenac sodium turned out to be ineffective (30). However, the systemic action of antibiotics observed after the administration of traditional oral dosage forms may be associated with side effects. Therefore, in the case of inflammatory conditions like colitis, the application of the dosage form releasing the drug at least partially in the colon could be beneficial in terms of therapeutic efficacy and safety.

Taking into account the processes related to inactivation of ROX, as well as the advantageous properties of gellan gum, the aim of the presented work was to obtain and characterize in detail the properties of novel polymer-based matrices with an incorporated drug as potential dosage forms. Considering the possible modification of drug release and $\mathrm{pH}$-resistant properties of the obtained 
systems, special attention has been paid to the impact exerted by the addition of other naturally derived polymers on the behavior and potential applicability of novel drug delivery systems.

\section{EXPERIMENTAL}

\section{Materials}

Roxithromycin Lot 0000020985 was purchased from Polfa Tarchomin S.A. (Warsaw, Poland). Tween ${ }^{\circledR} 80$ Lot BCBK6689V, gellan LA; Phytagel $^{\mathrm{TM}}$ Lot SLBT8352, Karaya gum, Lot SLBP5629V, Pectin from citrus peel Lot SLBN9007V, א-Carrageenan Lot BCBR6980V, Methylcellulose Lot SLBN3595, alginate Na Lot MKBL7997V were purchased from Sigma-Aldrich (St. Louis, USA). Calcium chloride Lot 0118/02/11 and potassium dihydrophosphate Lot 0115/01/16 were purchased from Avantor Performace Materials S.A. (Gliwice, Poland). Phosphate buffer $\mathrm{pH}=7.4$ concentrate Lot 18/03/02 was purchased from Firma Chempur $^{\circledR}$ (Piekary Śląskie, Poland). Hydrochloric Acid 0.1N, pH 1.2, DILUT-IT ${ }^{\mathrm{TM}}$ Dissolution media concentrate Lot 1628001868 (JT Baker) and phosphate buffer $\mathrm{pH}=4.5$ DILUT-ITTM Dissolution media concentrate Lot 1732801861 (JT Baker), acetonitrile HPLC isocratic grade Lot 1516913003 (JT Baker) and methanol HPLC isocratic grade Lot 1510026010 were purchased from Witko Sp. z o.o. (Łódź, Poland). Roxithromycin commercial tablets Rulid $150 \mathrm{mg}$ Lot 6AU7E (Sanofi-Aventis Sp. z o.o., Warsaw, Poland) were purchased off the shelf from a local drug store. Deionized water used for production of the matrices was prepared by the Simplicity ${ }^{\circledR}$ Water Purification System (Merck Millipore, Darmstadt, Germany).

\section{Methods \\ Composition and preparation of ROX-loaded gel- lan matrices}

The matrices were obtained by means of the ionotropic gelation technique. In the first step, the polymer, drug and surfactant mixtures were prepared. The percent amounts of the ingredients used for the production of six mixtures (R1-R6) are presented in Table 1. Initially, the polymer or polymers were sprinkled on the surface of deionized water (ambient temp.) in Erlenmeyer flasks and left to moisten and swell for $15 \mathrm{~min}$. The flasks were closed with a rubber septum to prevent water evaporation. During the next $15 \mathrm{~min}$, the temperature was raised to $80.0 \pm 2.0^{\circ} \mathrm{C}$ under constant stirring on RET control-visc magnetic stirrer (IKA, Staufen, Germany). When clear polymer solutions were obtained, the temperature was decreased to $70 \pm 2.0^{\circ} \mathrm{C}$ and Tween ${ }^{\circledR} 80$ was added. After 2 min of mixing ROX was carefully added and the mixing was continued until homogenous white suspensions were obtained. The mixtures were taken out from the flask using a $20 \mathrm{~mL}$ syringe. The syringes were placed in NE-1000 syringe pump (New Era Pump Systems, Inc., USA). The drug-polymer mixtures were transferred to the solution of calcium chloride $\left(9.0 \times 10^{-2} \mathrm{M}\right)$ through Var J1 encapsulation unit (NISCO Engineering AG, Zurich, Switzerland). The airflow through the encapsulator was set at $150 \mathrm{~L} / \mathrm{h}$. The medium $(60 \mathrm{~mL})$ was constantly mixed in a beaker. The temperature of the medium was ambient. After $15 \mathrm{~min}$ (curing time) the matrices were drained off through a sieve and washed threefold with deionized water to remove the excess of $\mathrm{CaCl}_{2}$. Further, the matrices were placed on glass Petri dishes and dried at $40.0 \pm 2.0^{\circ} \mathrm{C}$ for $48 \mathrm{~h}$ in ING 400 heater with the gravitational air circulation (Memmert GmbH, Germany). The dried matrices were stored in closed propylene string bags at ambient temperature with limited access to light.

\section{Stereoscopic microscopy of the wet matrices}

The wet matrices, directly after production were subjected to visual observation with the use of Discovery.V20 SteREO optical stereoscopic microscope (Zeiss, Switzerland) under the magnification of $90 x$.

Table 1. The percent compositions of the initial mixtures used for production of the matrices.

\begin{tabular}{|c|c|c|c|c|c|c|}
\hline Formulation & Gellan & ROX & Tween ${ }^{\circledR} 80$ & $\mathrm{H}_{2} \mathrm{O}$ & \multicolumn{2}{|c|}{ Additional polymer } \\
\hline R1 & 1.5 & 1.5 & 0.37 & 96.63 & \multicolumn{2}{|c|}{-} \\
\hline R2 & 1.0 & 1.5 & 0.37 & 96.63 & alginate Na & 0.5 \\
\hline R3 & 1.0 & 1.5 & 0.37 & 96.63 & pectin \\
\hline R4 & 1.0 & 1.5 & 0.37 & 96.63 & karaya gum & 0.5 \\
\hline R5 & 1.0 & 1.5 & 0.37 & 96.63 & methylcellulose & 0.5 \\
\hline R6 & 1.0 & 1.5 & 0.37 & 96.63 & $\kappa$-carageenan & 0.5 \\
\hline
\end{tabular}




\section{Scanning Electron Microscopy (SEM) of the dried matrices}

ROX powder and dry matrices were fixed to a stand using double-sided adhesive tape. To obtain the electrical conductivity, the samples were coated with a gold layer under vacuum in the Q150RS sputter coater (Quorum Technologies, UK). The samples were examined under the magnification of 100x and 2500x by a scanning electron microscope Vega3 LMU (Tescan, Czech Republic) equipped with tungsten electron emitter. The images were obtained under vacuum (25Pa) with the use of LVSTD detector.

\section{Raman analysis}

The non-polarized Raman spectra were recorded in the backscattering geometry using inVia Renishaw micro-Raman system (Renishaw, New Mills, UK). The Raman scattering spectra were investigated in the spectral range of $100-3200 \mathrm{~cm}^{-1}$. An infrared solid-state laser operating at $785 \mathrm{~nm}$ was used as a source of exciting light. The laser beam was tightly focused on the surface of the sample through Leica 50× LWD microscope with long working distance objective (LWD) (Leica, Wetzlar, Germany), with a numerical aperture (NA) equal to 0.5 . The laser beam diameter was equal to $2 \mu \mathrm{m}$. The air-cooled CCD camera detector (Rencam) and 1800 lines/mm diffraction grating were used. To prevent any damage of the sample an excitation power was fixed at no more than $10 \mathrm{~mW}$. The samples were studied at room temperature (296 K). The Raman scattering spectra for NPX powder, gellan gum and all dried matrices were obtained in the same spectral conditions (temperature, laser power, scattering geometry).

\section{Differential scanning calorimetry (DSC)}

DSC curves were obtained using a DSC XP-10 differential scanning calorimeter (Haas, Poland). Samples (2-6 mg) were placed in a standard $40 \mu \mathrm{L}$ aluminum pans with pierced lids and heated with $10^{\circ} \mathrm{C} \mathrm{m^{-1 }}$ rate from 40 to $180^{\circ} \mathrm{C}$ under a nitrogen gas flow of $20 \mathrm{~mL} \mathrm{~min}^{-1}$. An empty pan was used as a reference.

\section{Swelling behavior}

The dried matrices were investigated toward the tendency to absorb water at different $\mathrm{pH}$ values. Three acceptor fluids were used, namely $0.1 \mathrm{M}$ hydrochloric acid solution ( $\mathrm{pH}=1.2$ ), acetate buffer $(\mathrm{pH}=4.5)$ and phosphate buffer $(\mathrm{pH}=7.4)$. Initially, approximately $30.0 \pm 0.1 \mathrm{mg}$ of the matrices were placed in the beakers $(25 \mathrm{~mL})$ and immersed in $10.0 \mathrm{~mL}$ of the fluid (previously heated to $37.0 \pm$ $\left.0.5^{\circ} \mathrm{C}\right)$. Then the beakers were fixed to $\mathrm{KS} 130$
Control orbital shaker (IKA ${ }^{\circledR}$, Staufen, Germany), located in BD 30 heater with the gravitational air circulation (Binder, Gleisdorf, Austria) and mixed at $150 \mathrm{rpm}$. After certain periods (1, 2, 3 and $6 \mathrm{~h}$ ) the matrices were removed from the fluid, gently blotted up with the paper towel and weighed. After weighing, the matrices were immersed in the fresh portion of the fluid. The swelling index (SI) was calculated using equation 1 :

$$
\% \mathrm{SI}=\frac{\mathrm{W} 2-\mathrm{W} 1}{\mathrm{~W} 1} \times 100
$$

where W1 is the weight of dry matrices and W2 is the weight of the swollen matrices.

\section{Determination of ROX content and loading effi- ciency into the matrices}

The dried matrices were weighed in the amounts equal to theoretical $10.0 \mathrm{mg}$ of ROX, poured to glass volumetric flasks and immersed in $25 \mathrm{~mL}$ of methanol in the closed glass flasks. The flasks were placed on the orbital shaker (KS 130 Control; IKA ${ }^{\circledR}$ ) in the heater (BD 30, Binder, Gleisdorf, Austria). The temperature was set at 37.0 $\pm 0.5^{\circ} \mathrm{C}$. After $24 \mathrm{~h} 1.0 \mathrm{~mL}$ of the solution was taken from the flask, diluted three-fold with methanol and subjected to chromatographic analysis with the use of LC-10AT VP chromatograph connected with UV-Vis SPD-10A VP spectrophotometer (Shimadzu, Japan). The assay was performed on ProntoSIL 120-5-C18 H, $5 \mu \mathrm{m}, 150$ x 4.6 mm chromatographic column (Bischoff Chromatography, Germany). The mobile phase consisted of acetonitrile and phosphate buffer $(\mathrm{pH}=6.0)$ at $65: 35(v / v)$ ratio. The assay was conducted under isocratic conditions and the flow rate was equal to $1.2 \mathrm{~mL} / \mathrm{min}$. The column was placed in the thermostat adjusted to $40.0 \pm$ $2.0^{\circ} \mathrm{C}$. The analytical wavelength was $206 \mathrm{~nm}$. The drug loading and drug encapsulation efficiency were calculated according to using equations 2 and $3(31)$ :

Drug loading $(\%)=\frac{\text { amount of NPX in the matrices }}{\text { mass of the matrices }} \times 100$ (Eq. 2)

NPX encapsulation efficfiency $(\%)=\frac{\text { actual NPX content in matrices }}{\text { theoretical drug content in matrices }} \times 100$ (Eq. 3)

\section{In vitro drug release studies}

The drug release studies were performed on USP 1 apparatus - rotating basket (Kraemer Elektronik GmbH, Germany). The test was performed separately for $\mathrm{pH}=1.2$ and 7.4 in the volume of $500.0 \mathrm{~mL}$. The temperature was set at $37.0 \pm$ $0.5^{\circ} \mathrm{C}$. The weighed samples $(\sim 100.0 \mathrm{mg})$ of the matrices were closed in gelatin capsules, which were placed in the baskets and immersed in a prop- 
er fluid. The total time of the experiment was 60 min. The rotation speed of the baskets was set at 100 $\mathrm{rpm}$. After certain periods $1.0 \mathrm{~mL}$ of the fluid was withdrawn from the vessel, replaced with the same volume of the fresh fluid and further subjected to HPLC assay according to the conditions given in the previous paragraph.

\section{Statistical analysis}

The statistical analyses were performed with Statistica ver.13 software (32). The results obtained for the investigated formulations were subjected to one-way analysis of variance (ANOVA). For the comparison of the mean values of the analyzed parameters post-hoc Scheffe's test was used. In all performed tests the significance level was set at $5 \%$.

\section{RESULTS AND DISCUSSION}

The shape, size, internal structure and mechanical properties (elasticity, hardness) of the gellan matrices obtained by ionotropic gelation depend on many various factors (33). The most important ones are concentration and type of the polymer, physicochemical properties of the drug or additives and type of the crosslinking ions. The other important parameters are the conditions during the production process i.e. temperature, flow rate, diameters of the tubing and needle, height of instillation, curing time, etc. (33-36). In the presented work the main goal was to investigate the effects of blending gellan with other naturally derived polymers mostly in relation to their predicted behavior in the GIT. All other

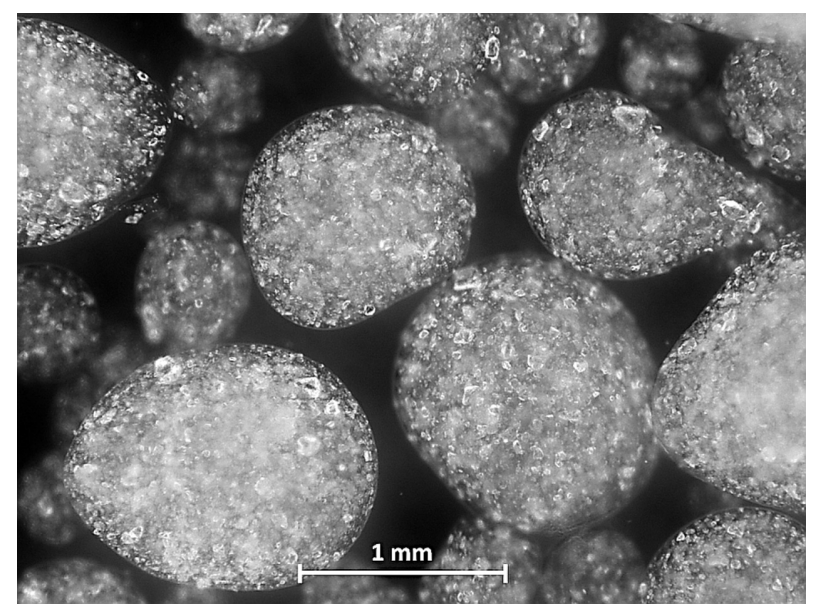

Figure 2. The R5 matrices in water before drying.

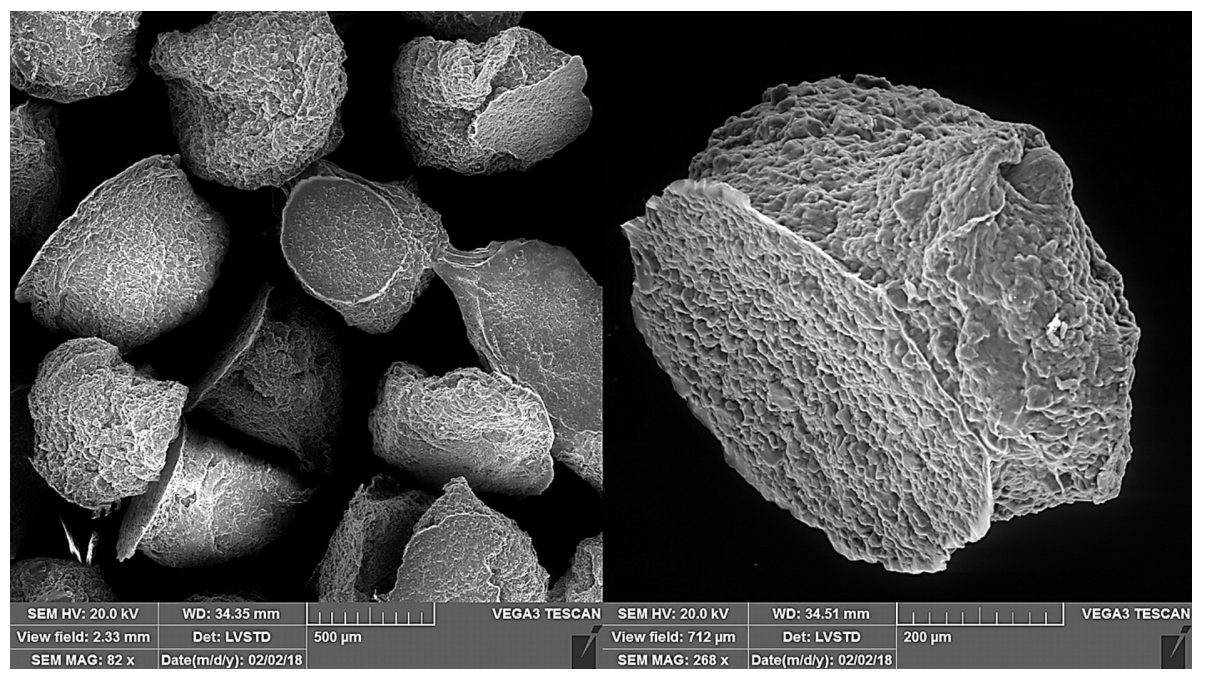

Figure 3. SEM image of matrices R5 after drying. 
components were used at constant amounts and the conditions during the production process were also identical for all formulations. Another aspect was to determine whether gellan matrices can act as protectors for ROX against stomach acidic environment and/or influence its release pattern in distal parts of GIT.

Morphology of the wet matrices and after drying

Directly after production, the matrices were subjected to visual observation with the use of an optical microscope. The suspensions of the matrices in water were poured on plastic Petri dishes and observed under the magnification of 90x. As can be seen in Figure 2, the matrices differed in shape and size. Most of them were spherical but also oval and comma shapes were observed. The obtained images suggest the presence of the drug crystals at the matrices surface, however, taking into consideration the manufacturing process it may be expected that the active ingredient is dispersed in the whole volume of the matrices.

Next, the dried matrices were investigated with the use of SEM microscopy. As can be seen in Figure 3, the matrices were not spherical and in most cases the flattening located on one side was present. Such deformation occurred due to the loss of elas- ticity of the matrices during water evaporation. The surface of all matrices was irregular and wrinkled. It can be also observed that drug crystals partially bulged out of the polymer matrix. Moreover, it was revealed that the matrices had the tendency to aggregate during a water loss. The effects were most visible in the case of formulations R2 and R3. In the case of R3 matrices, water loss contributed to collapsing of the polymer network and as a result, their shape was similar to flakes.

\section{Raman analysis}

As can be seen in Figure 4, in the case of all samples, besides pure polymers the characteristic band for ROX was observed at the wavelength of $1726 \mathrm{~cm}^{-1}$. The band is probably related to the presence of the carbonyl moiety. Its occurrence or partial fading could be the result of various drug content in the matrices or the effect of strong Rayleigh scattering (high background) which was covering the Raman signal. In all spectra, the bands related to - $\mathrm{CH}$ group were observed in the range of 2800-3000 $\mathrm{cm}^{-1}$. At such high values, no Rayleigh scattering is possible, there the band could be attributed to ROX, however, a signal from other components could not be neglected. No signs of drug decomposition were observed.
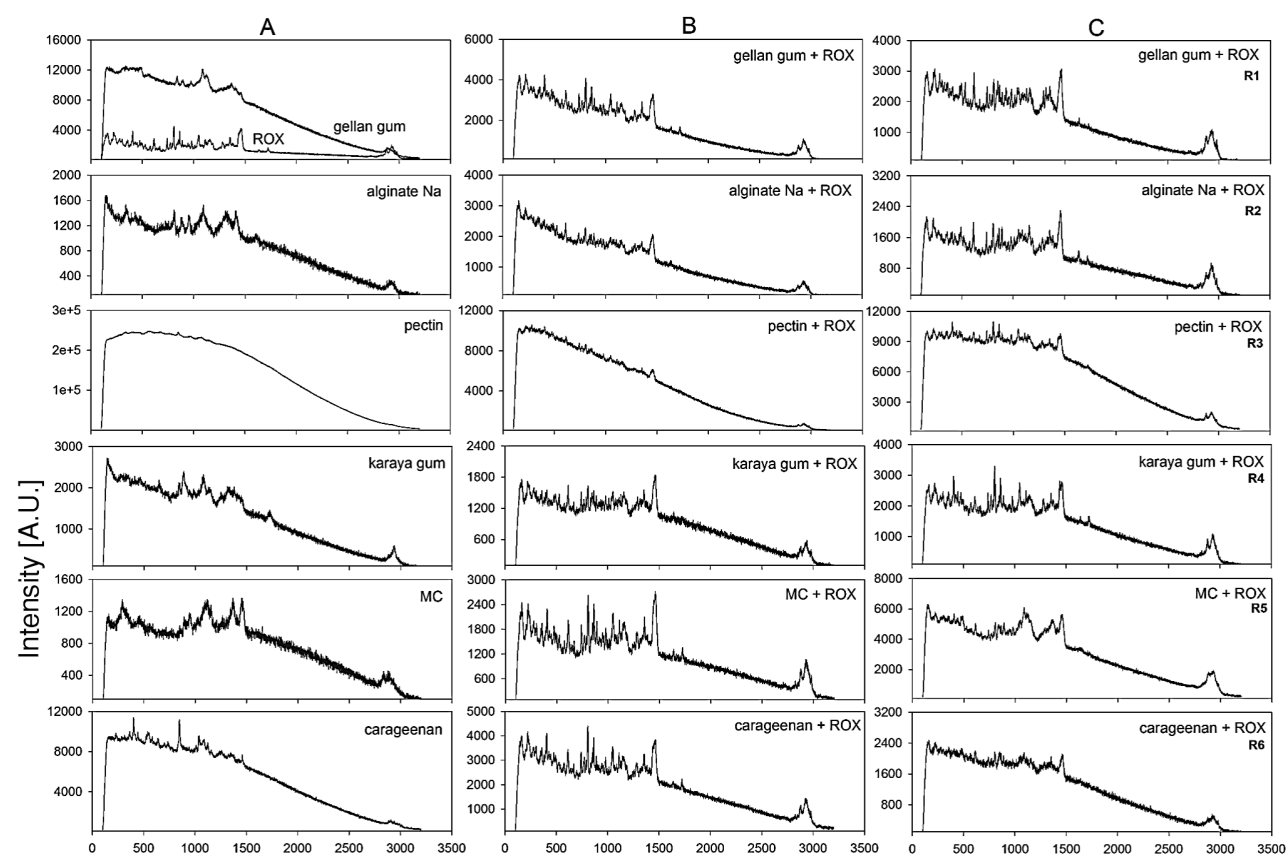

Wave number $\left[\mathrm{cm}^{-1}\right]$

Figure 4. Raman spectra of pure components (A), physical mixtures of polymers and ROX (B), and dried matrices (C). 


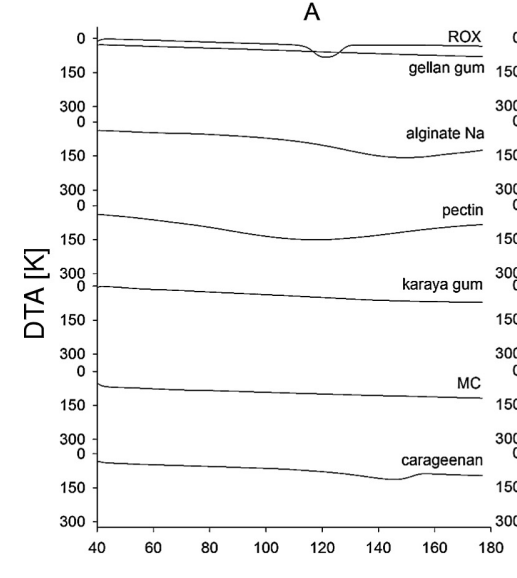

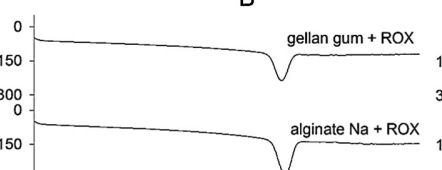

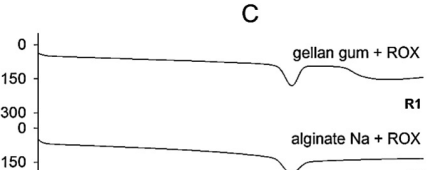

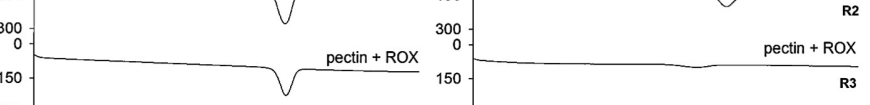
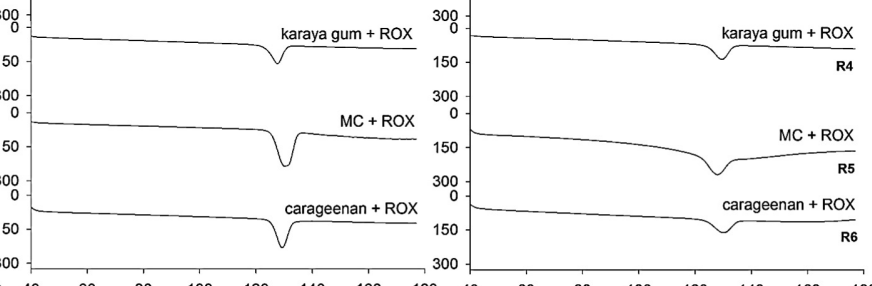

Temperature $\left[{ }^{\circ} \mathrm{C}\right]$

Figure 5. The DSC curves obtained for pure components (A), physical mixtures of ROX and polymers (B), dried matrices (C).

\section{Differential scanning calorimetry (DSC)}

The DSC experiments performed for pure drug revealed endothermic peak corresponding to melting at about $120^{\circ} \mathrm{C}$ (Fig. 5A) which is similar to the values presented in the literature reports (37-39). The obtained results indicate that the investigated drug occurs in the crystalline state. In the case of pure polymer samples, no melting processes were observed. Diffuse peaks recorded in thermograms of sodium alginate, pectin and carrageenan are most probably related to water evaporation. Therefore, it may be assumed that all polymers applied in matrices formation occur in an amorphous state. In the thermograms obtained for the physical mixtures of ROX and different polymers peaks attributed to the drug melting were observed (Fig 5B). It is noteworthy that in all cases the peaks were shifted to higher temperatures when compared to the pure drug. The same effect was observed by Biradar et al. for the physical mixtures containing ROX with poloxamer 407, hydroxypropyl methylcellulose, sodium carboxymethylcellulose, lactose monohydrate and soybean lecithin (40). Melting processes were also observed for the dried matrices (Fig. 5C) with additional components, i.e. gellan, sodium alginate, karaya gum, MC and carrageenan. Again, melting points of the mentioned systems were higher in comparison to the pure ROX. This may indicate the possible interaction between the matrices components. In the matrices containing pectin, the peak related to ROX melting was almost indiscernible which could be explained by the partial occurrence of the drug in an amorphous state. The observed phenomenon is important in terms of the drug disso- lution in the gastrointestinal tract. Substances occurring in an amorphous form are dissolved and released faster than those administered in a crystalline state. The described differences in the dissolution rate are particularly important for the substances revealing poor water solubility and may significantly contribute to the observed therapeutic effect. However, according to Aucamp et al. an amorphous form of ROX undergoes a glass transition at about $77^{\circ} \mathrm{C}$ which was not observed in our study performed for the matrices with pectin. Moreover, an additional thermal effect was observed above $150^{\circ} \mathrm{C}$ for $\mathrm{R} 1$ matrices containing gellan gum only (41). It may be assumed that the recorded diffuse peak corresponds to the decomposition process. The obtained result indicates that the stability of R1 matrices may be affected by high temperatures which is important information in the design of the manufacturing process.

\section{Swelling behavior}

The swelling behavior was conducted in order to determine how and to what extent the matrices absorb water at various $\mathrm{pH}$. The experiment was performed in three fluids, each adjusted to different $\mathrm{pH}$ corresponding to the physiological conditions, namely $1.2 ; 4.5$ and 7.4. The results are presented in Figure 6. As can be seen, the matrices absorbed water in all fluids, however with various rates and following various patterns. The slowest changes were observed at $\mathrm{pH}=1.2$. In general, the mass was increasing during the first hour and after that, no apparent changes were occurring. The highest mass increase was noticed for formulation R4 (197.4\%), 
which consisted of gellan/karaya gum blend. It must be taken into account that the mass changes in the lowest $\mathrm{pH}$ could not only be the result of water absorption. Due to irregular surface of the matrices water could also remain in the folds or cavities and it was not able to entirely remove it despite very careful blotting. Additionally, it was observed that the matrices became transparent at the end of the test. According to expectations, at higher $\mathrm{pH}$ values water absorption was much more efficient. However, surprisingly swelling to the highest extent was observed at $\mathrm{pH}=4.5$. As can be seen in Figure 6 the swelling was most rapid up to the second hour. The mass was increasing until the end of the experiment and the highest value $(\sim 1760 \%)$ was reached by matrices R5 (methylcellulose). The matrices did not lose their shape and elasticity. The exceptions were formulations R1 (gellan) and R3 (gellan+pectin) which showed the evidence of polymer network decomposition, starting from the third hour. It can be supposed that very efficient swelling at $\mathrm{pH}=4.5$ can be the result of acetate ions that contributed to the reduction of the $\mathrm{Ca}^{2+}$ crosslinking

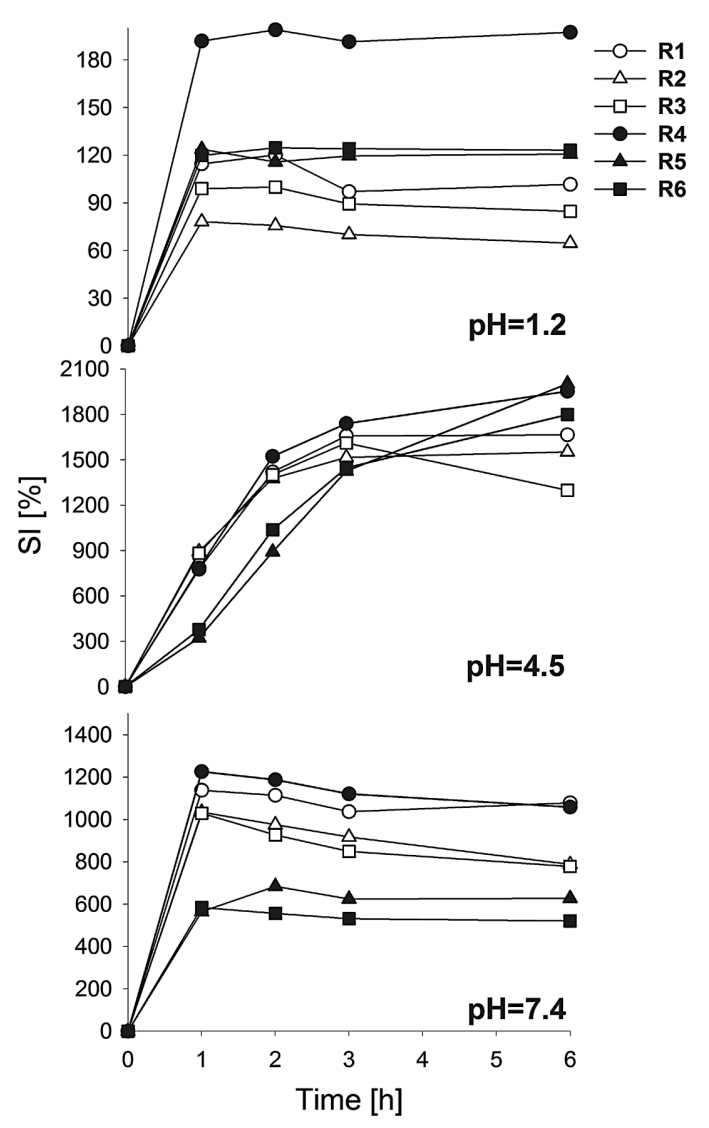

Figure 6. Swelling of the dried matrices in three different media. bridges, which caused the polymer network to be more susceptible to the penetration by water. It also has to be kept in mind that in physiological conditions the formulation will be surrounded by such conditions for the time of 15-30 min which means that from the practical point of view the observed effect is less significant than the results obtained at the other $\mathrm{pH}$ values. The matrices swelled according to a different pattern in $\mathrm{pH}=7.4$. During the first hour, the swelling was rapid and after that time the mass loss was observed. This can be attributed to the decomposition of the polymer network and the release of the drug. Both processes were equilibrated by absorption of water, therefore the mass of the matrices did not change distinctly. It can be also observed that two formulations, namely R5 (methylcellulose) and R6 ( $\kappa$-carrageenan), revealed a weaker tendency to absorb water. Probably this was due to greater rigidity of the polymer network. As was mentioned before, these two formulations did not deform and flatten distinctly during drying, which could be the evidence of higher mechanical resistance and hardness. It may be assumed that because of matrix properties water did not penetrate the matrix easily.

\section{Determination of ROX encapsulation efficiency and content in the matrices}

In general, it can be stated that encapsulation of ROX into the matrices proceeded with high efficiency. As can be observed in Figure 7 the drug was entirely encapsulated $(101.5 \pm 0.7 \%)$ in matrices R1, composed of pure gellan. In the case of other formulations, the smallest amount of ROX (86.9 \pm $0.0 \%$ ) was encapsulated in matrices $\mathrm{R} 4$, containing gellan and karaya gum. According to the results, it can be assumed that no significant drug decomposition occurred during the production process.

\section{Drug release studies}

At first, the investigated formulations were subjected to the drug release study for enteric dosage forms described in European Pharmacopoeia. To follow the protocol, the enteric products immersed in the acidic solution $(\mathrm{pH}=1.2)$ should stay resistant and release no more than $10 \%$ of the drug within $2 \mathrm{~h}$. Next, the experiment should be continued at higher $\mathrm{pH}$ values reflecting the physiological conditions in duodenum. Unfortunately, in the case of our matrices, it turned out to be difficult to accomplish the first stage of the test. ROX revealed very fast decomposition even though it was encapsulated in the polymer network. After two hours of the experiment conducted at acidic $\mathrm{pH}$ no signal from the drug was detected. 


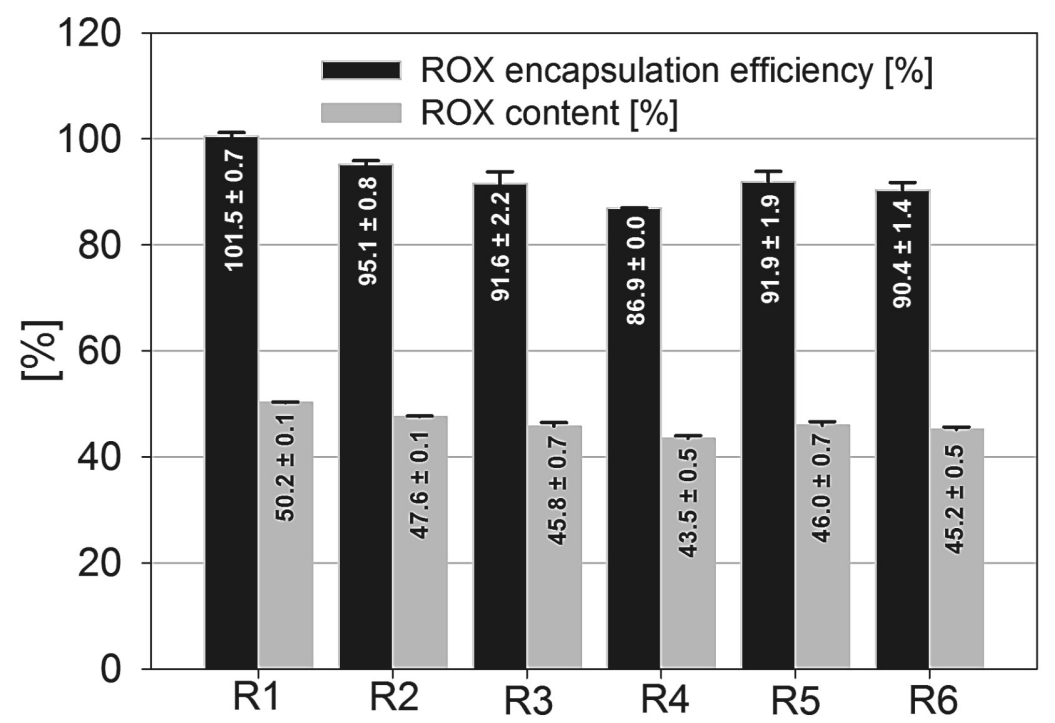

Figure 7. ROX encapsulation efficiency and content in the obtained matrices.
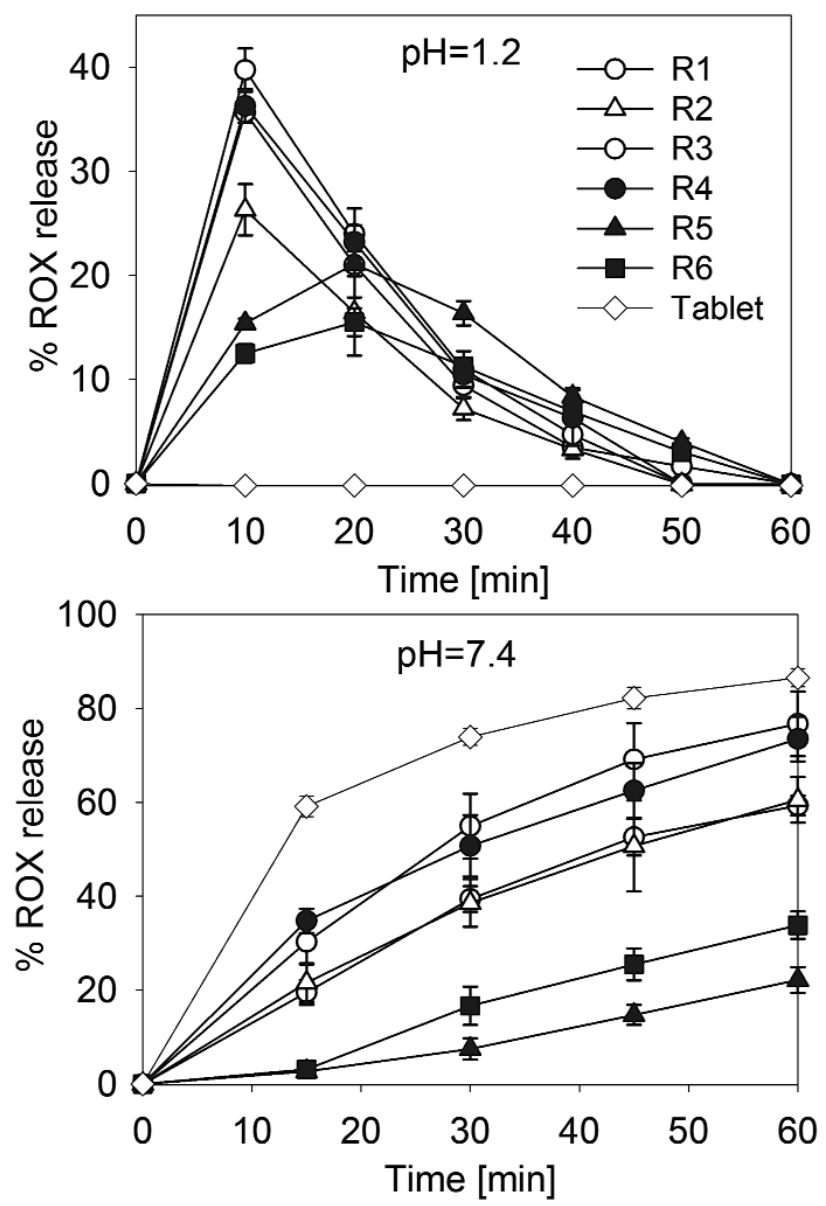

Figure 8. ROX release profiles from matrices and commercial tablets obtained separately at $\mathrm{pH}=1.2$ and 7.4. 
Therefore, we have decided to conduct separate studies for $\mathrm{pH}=1.2$ and 7.4. The aim was to investigate whether the polymer composition of the matrix has an influence on the drug release.

Each study was performed for $60 \mathrm{~min}$. As can be seen in Figure 8, in the acidic conditions the drug decomposed in the case of all investigated polymer matrices. It was observed that in 10 min the concentration of the drug in the receptor medium increased, however, at the same moment the drug started to decompose and its concentration gradually decreased until it reached zero value after $60 \mathrm{~min}$. Taking into consideration the results obtained for the matrices with different additional polymers, it may be assumed that the highest ROX concentration in the acceptor medium was recorded in the case of R3 (pectin) and the lowest one was observed for R6 (carrageenan). Moreover, the visual inspection of the matrices during the experiment revealed that their shape did not change. However, it was also observed that in the course of study, the matrices got discolored. The observed processes indicate that even though the matrices were stable in the acidic environment, they were not able to protect the encapsulated drug. It is noteworthy that this effect was independent of the matrix composition. The inability to protect the drug from degradation may be explained by the gradual decomposition of ROX crystals and the formation of pores enabling further penetration of the acidic solution into the matrices. In the images obtained with SEM (Fig. 2 and 3) the drug crystals at the surface of the matrices are visible which means that some part of the active ingredient is exposed directly to the acidic environment and decomposes instantly after the immersion.

As a result, the crystals at the bead surface are replaced with open spaces through which the acceptor medium can penetrate deeper regions. Therefore, the crystals located in further layers of the bead are also subjected to the destructive action of the acidic medium. To the best of our knowledge, the studies focusing on the encapsulation of the drugs sensitive to the physiological conditions in gellan matrices are quite rare. Meneguin et al. studied the matrices with insulin which requires protection from digestive enzymes present in the gastrointestinal tract. They obtained gellan matrices with the ability to effectively protect the drug from the degradation process, however, in this study, the matrices were coated with resistant starch/pectin films. The presented method could be also effective in the case of acid-sensitive drugs (42). Another observations were made in the experiment conducted at $\mathrm{pH}=7.4$. All investigated formulations released the drug instantly. However, some statistically significant differences related to the rate of the process and the amount of the drug released after 60 min were observed. Differences visible in the drug release profiles revealed that the modification of the matrix composition with the addition of other polymers may affect the drug release in the intestines. Depending on the applied polymer the amount of the released drug varied from about $20 \%$ to about $70 \%$ of the initial drug content. It was also noted that the drug release rate was the lowest for R5 and R6 which corresponds to the considerations related to their shape and hardness. The highest amount of ROX was released from R3 containing pectin as an additional polymer.

The obtained formulations were also considered as potential modified-release dosage forms designed for shifting the drug release process to the distal parts of the GI tract. Taking into account the drug release differences at $\mathrm{pH}=7.4$ it may be assumed that matrix composition had an impact on the potential applicability of the obtained products. In the case of formulations R5 and R6, only about $20-30 \%$ of the drug was released after 60 min of the experiment which is promising in terms of moving the remaining amount of the active ingredient to distal parts of the GI tract. It is noteworthy that the observed effects correlate with the results of swelling experiments. The drug release rate was lower for the matrices revealing also the weakest swelling ability which is most probably related to the slower penetration of water to the internal regions of the matrices. It is also noteworthy that both mentioned formulations behaved differently from the other formulations in the drug release tests performed at $\mathrm{pH}=1.2$. Despite the fact that in all experiments the drug was finally decomposed, in the case of formulations R5 and R6 the obtained drug release profiles show that the initial ROX concentrations were lower. Nevertheless, the observed effects related to the instability of the active ingredient indicate that some technological improvements have to be introduced in order to obtain efficient and stable dosage form with the ability to release the drug also in the distal parts of the GI tract.

\section{CONCLUSIONS}

Due to the unique structure and beneficial properties, gellan gum is widely described and investigated as a potential multifunctional component for various pharmaceutical products. The ion sensitivity and specific gelling properties have led to the development of controlled-release forms. Numerous formulations based both on high-acyl and low- 
acyl gellan have been studied, including oral, ophthalmic, nasal, rectal and other delivery routes. In the presented work we have focused on the design and evaluation of low-acyl gellan gum matrices containing acid-sensitive drug, roxithromycin. The aim was to prove or deny whether the polymer can provide protection for the drug during the passage through the acidic environment of the stomach. Another aspect was to define the influence of other natural polymers on the $\mathrm{pH}$-sensitive matrices behavior in simulated gastrointestinal conditions. The described experiments clearly fit in the preformulation stage of novel drug delivery systems development. Undoubtedly, the results can be the source of valuable information and completion of the general knowledge in terms of the pharmaceutical application of gellan.

The obtained results have shown that besides the stability of the matrices at low $\mathrm{pH}$ they did not protect the encapsulated drug, independently of the matrix composition. This was probably due to the fact, that the drug crystals were located inside the matrices but also attached to their surface or only partially immersed. It may be assumed that the crystals from the surface dissolved and decomposed and thus left open spaces for the fluid to penetrate the polymer network. As a result, the matrices did not change their shape and volume, but the drug was rinsed out, which led to the transformation of the entire dose within the time of $60 \mathrm{~min}$.

On the other hand, interesting results were obtained when the matrices were immersed at $\mathrm{pH}=$ 7.4. The drug release experiments clearly showed that by blending of gellan with other natural polymers the release pattern can be changed distinctly which might be promising for shifting the drug release to the distal parts of the GI tract. The best results in terms of the drug release modification were observed for the matrices containing gellan / methylcellulose and gellan / $\kappa$-carrageenan mixtures. It is noteworthy that the observed drug release profiles correspond to the swelling behavior recorded for the investigated formulations. The lowest drug concentration after $60 \mathrm{~min}$ at $\mathrm{pH}=7.4$ was related to the weakest swelling ability which might explain the slower water penetration to the internal parts of the matrices and also slower drug diffusion. The results of the study indicate clearly that the investigated complex polymer matrices show could be considered as novel modified-release dosage forms with potential ability to release the drug at least partially in the colon which may be advantageous in the treatment of the inflammatory conditions localized in the distal parts of GI tract.
However, it was also shown that the investigated technology requires further modifications in order to protect the acid-sensitive active ingredient from chemical degradation.

\section{Acknowledgments}

The work was financed by the National Science Centre (Poland) grant No. DEC2017/01/X/NZ27/00544 (grant ID: 370652). The authors would like to thank Professor Artur Bartkowiak from the Center of Bioimmobilisation and Innovative Packaging Materials, West Pomeranian University of Technology (Szczecin, POLAND) for the access to the encapsulation equipment, scientific support and advisory. We are also very grateful to Professor Janina Lulek from the Department of Pharmaceutical Technology, Poznan University of Medical Sciences (Poznań, POLAND) for support and scientific advisory.

\section{Conflict of interests}

The authors declare no conflict of interest.

\section{REFERENCES}

1. Debotton N., Dahan A.: Med. Res. Rev. 37, 52 (2017).

2. Dang J.M., Kam W.L.: Adv. Drug Deliver. Rev. 58, 487 (2006).

3. Pillali O., Panchagnula R.: Curr. Opin. Chem. Biol. 5, 447 (2001).

4. Schmaljohann D.: Adv. Drug Deliver. Rev. 58, 1655 (2006).

5. Ravi V., Kumar T.M.P., Siddaramaiah: Ind. J. Pharm. Sci. 70, 111 (2008).

6. Erah P.O., Goddard A.F., Barrett D.A., Shaw P.N., Spiller R.C.: J. Antimicrob. Chemother. 39, 5 (1997).

7. Sun J., Zhang T., Qiu F., Liu Y., Tang J., et al.: J. Antimicrob. Chemother. 55, 796 (2005).

8. Blessy M.R.D.P., Patel R.D., Prajapati P.N., Agrawal Y.K.: J. Pharm. Anal. 4, 159 (2014).

9. Ostrowski M., Wilkowska E., Bączek T.: Cent. Eur. J. Med. 5, 83 (2010).

10. Iuga C., Bojiţă M.: Farmacia 59, 203 (2010).

11. Amidon S., Brown J.E., Dave V.S.: AAPS PharmSciTech 16, 731 (2015).

12. Inukai K., Noguchi S., Kimura S.I., Itai S., Iwao Y.: J. Pharm. Sci. 107, 2514 (2018).

13. De Robertis S., Bonferoni M.C., Elviri L., Sandri G., Caramella C., Bettini R.: Exp. Opin. Drug Deliver. 12, 441 (2015). 
14. Agüero L., Zaldivar-Silva D., Peńa L., Dias M.L.: Carbohydr. Polym. 168, 32 (2017).

15. Gadalla S.H.H., Soliman G.M., Mohammed F.A., El-Sayed A.M.: Drug Deliver. 23, 2541 (2016).

16. Pushpamalar J., Veeramachineni A.K., Owh C., Loh X.J.: ChemPlusChem 81, 504 (2016).

17. Boni F.I., Prezotti F.G., Cury B.S.F.: Drug Dev. Ind. Pharm. 42, 1283 (2016).

18. Günter E.A., Markov P.A., Melekhin A.K., Belozerov V.S., Martinson E.A., et al.: Int. J. Biol. Macromol. 120, 2225 (2018).

19. Yoshida T., Shakushiro K., Sako K.: Curr. Drug Targets 19, 225 (2018).

20. Patil S., Sharma S., Nimbalkar A., Pawar A.: Drug Dev. Ind. Pharm. 32, 315 (2006).

21. Patil P., Chavanke D., Wagh M.: Int. J. Pharm. Pharm. Sci. 4, 27 (2012).

22. Osmałek T., Milanowski B., Froelich A., Szybowicz M., Białowąs W.: Drug Dev. Ind. Pharm. 43, 1314 (2017).

23. Shafuddin E., Mills G.D., Holmes M.D., Poole P.J., Mullins P.R., Black P.N.: J. Neg. Res. Biomed. 14, 15 (2015).

24. Young R.A., Gonzalez J.P., Sorkin E.M.: Drugs 37, 8 (1989).

25. Shuqiu Z., Xing J., Zhong D.: J. Pharm. Sci. 93, 1300 (2004).

26. Kwiecień A., Krzek J., Żmudzki P., Matoga U., Długosz M.: Anal. Met. 6, 6414 (2014).

27. Koopaei M.N., Maghazei M.S., Mostafavi S.H., Jamalifar H., Samadi N., et al.: DARU 20, 92 (2012).

28. Agen C., Danesi R., Blandizzi C., Costa M., Stacchini B., et al.: Agent. Act. 38, 85 (1993).
29. Choi Y., Koh S.-J., Lee H.S., Kim J.W., Kim B.G., et al.: Exp. Biol. Med. 240, 1664 (2015).

30. Ahmad H., Verma S., Kumar V.L.: Inflamm. Res. 67, 147 (2017).

31. Kumar N.A., Pal D.: Int. J. Biol. Macromol. 49, 784 (2011).

32. Dell Inc. Dell Statistica (data analysis software system), version 13. software.dell.com (2016, accessed 8 July 2019).

33. Prezotti F.G., Cury B.S.F., Evangelista R.C.: Carbohydr. Polym. 113, 286 (2014).

34. Babu J., Sathigari S., Kumar M., Pandit J.K.: Curr. Drug Deliver. 7, 36 (2010).

35. Sunil A., Sheetal S., Tejraj M.: Eur. J. Pharm. Biopharm. 63, 249 (2006).

36. Alhaique F., Santucci E., Carafa M., Coviello T., Murtas E., Riccieri F.M.: Biomaterials 17, 1981 (1996).

37. More A.B., Chilgunde S.N., Kamble J.C., Patil P.S., Malshe V.C., et al.: J. Pharm. Sci. 98, 4781 (2009).

38. Gao Y., Cui F., Guan Y., Yang L., Wang Y., Zhang L.: Int. J. Pharm. 318, 62 (2006).

39. Yu J.P., Guan Y.X., Yao S.J., Zhu Z.Q.: Ind. Eng. Chem. Res. 50, 13813 (2011).

40. Biradar S.V., Patil A.R., Sudarsan G.V., Pokharkar V.B.: Powder Technol. 169, 22 (2006).

41. Aucamp M., Liebenberg W., Strydom S.J., van Tonder E.C., de Villiers M.M.: (AAPS PharmSciTech 13, 467 (2012).

42. Meneguin A.B., Beyssac E., Garrait G., Hsein H., Cury B.S.F.: Eur. J. Pharm. Biopharm. 123, 84 (2018).

(C) 2020 by Polish Pharmaceutical Society. This is an open-access article under the CC BY NC license (c) (1) (9) (http://creativecommons.org/licenses/by-nc/4.0/). 